\title{
EXPRESSION OF PROCOLLAGEN A1 TYPE I INDUCED BY TWO DIFFERENT DENTINE BONDING SYSTEMS IN HUMAN PULP FIBROBLASTS
}

\author{
G. ORSINI ${ }^{1}$, M. MATTIOLI-BELMONTE ${ }^{2}$, M. ORCIANI ${ }^{2}$, A. MAZZONI $^{3,4}$, G. TETI $^{5}$, \\ M. FALCONI ${ }^{5}$, A. PUTIGNANO ${ }^{1}$ and L. BRESCHI ${ }^{3,4}$ \\ 'Department of Clinical Sciences and Stomatology, Università Politecnica delle Marche, Ancona, \\ Italy; ${ }^{2}$ Department of Clinical and Molecular Sciences, Università Politecnica delle Marche, \\ Ancona, Italy; ${ }^{3}$ Department of Medical Sciences, Unit of Dental Sciences and Biomaterials, \\ University of Trieste, Trieste, Italy; ${ }^{4} I G M-C N R$, Unit of Bologna clo IOR, Bologna, Italy; \\ ${ }^{5}$ Department of Biomedical and Neuromotory Sciences, University of Bologna, Bologna, Italy
}

\section{Received December 5, 2012 - Accepted March 14, 2013}

This study aimed to evaluate the effects of two different dentine bonding systems (DBSs) on primary cultures of human pulp fibroblasts (HPFs). Cell viability and procollagen a1 type I expression were investigated. Polymerised resin disks of the bonding agent from a two-step self-etch system and of the primer/bonding agent from a two-step etch-and-rinse system were used to condition culture medium for 24 or $96 \mathrm{~h}$. HPFs were incubated in control (untreated) or DBSs-conditioned medium for $24 \mathrm{~h}$. HPF viability was determined using the 3-dimethylthiazol-2,5-diphenyltetrazolium bromide (MTT) assay. Western blot analysis was used to analyse procollagen $\alpha 1$ type I expression. Statistical analyses were performed using Student's $t$-tests. The results showed that HPFs incubated with DBSs-conditioned medium for $24 \mathrm{~h}$ demonstrated a significant reduction in the percentage of viable cells versus cells incubated with control medium (45\% for self-etch DBS and $30 \%$ for etch-and-rinse DBS; $p<0.05$ ), whereas this percentage increased significantly after exposure to the 96h DBSs-conditioned medium $(62 \%$ and $77 \%$, respectively; $p<0.05)$. Procollagen $\alpha 1$ type I expression in HPFs was strong for control specimens, but decreased in $24 \mathrm{~h}$-DBSs-conditioned medium, and was abolished with 96 h-DBSsconditioned medium. In conclusion, HPF exposure to medium containing eluates of the different DBSs led to an early cytotoxic effect $(24 \mathrm{~h}$ ) that decreased after a conditioning time of $96 \mathrm{~h}$, whereas procollagen $\alpha 1$ type I expression decreased at $24 \mathrm{~h}$ and was absent after $96 \mathrm{~h}$. Procollagen $\alpha 1$ type I expression may be a useful parameter for evaluating DBSs biocompatibility.

Dental pulp is a loose connective tissue, composed of fibroblasts (the most abundant cell population), odontoblasts, and undifferentiated mesenchymal cells that are in contact with a complex chain of macromolecules secreted in the extracellular matrix (ECM) (1). The pulp ECM is predominately composed of types I and III collagen fibrils (56\% and $41 \%$, respectively), in contrast to the predominantly type I collagen of dentine $(2,3)$. As a secretory protein, fibrous collagen is synthesized as procollagen and subsequently modified and assembled into the proper collagen triple helix (4). In vitro, fibroblasts produce

Key words: cell viability, dentine bonding systems, human pulp fibroblasts, procollagen al type I

Mailing address: Prof. Lorenzo Breschi,

Department of Medical Sciences,

1721-727X (2013)

University of Trieste,

Piazza Ospedale, 1,

I-34129 Trieste, Italy.

Tel.: +390403992192 Fax: +390403992665

e-mail: lbreschi@units.it reproduced without written permission from the copyright holder. Unauthorized reproduction may result in financial and other penalties DISCLOSURE: ALL AUTHORS REPORT NO CONFLICTS OF INTEREST RELEVANT TO THIS ARTICLE. 
procollagen $\alpha$ l type I in large amounts, and changes in procollagen $\alpha$ l type I protein expression were reported to be related to cytotoxicity (5). Most dental restorative procedures use resin-based materials associated with dentine bonding systems (DBSs). Several studies have reported that during DBS application to the dentine substrate, monomers can be released and reach the dental pulp through the dentinal tubules, causing adverse effects, such as dental pulp inflammation (6). In vitro, cytotoxicity of the resin monomers contained in DBSs has been evaluated previously using cell lines and/or primary fibroblasts obtained from pulp, gingiva, and periodontal ligaments (7-12). Reduced synthesis to inhibition of procollagen $\alpha 1$ type I synthesis has been demonstrated when human pulp fibroblasts (HPFs) were exposed to resinous monomers, such as 2-hydroxyethyl methacrylate (HEMA) (8). However, the direct effects of commercially available DBSs on procollagen $\alpha$ l type I expression has not been reported previously. Depletion of procollagen $1 \alpha 1$ type I synthesis could, in fact, impair HPF function, thus reducing their capacity to positively react to pulp injuries and differentiate into odontoblastic cells (13). Thus, the aim of the present study was to evaluate the possible toxic effects of commercially available DBSs on HPFs. The null hypotheses tested were that DBSs and the exposure time of DBSs in the culture medium would not affect i) HPF viability nor ii) procollagen al type I synthesis.

\section{MATERIALS AND METHODS}

\section{Establishment of human pulp fibroblast culture}

HPFs were isolated from third molars of healthy volunteers during routine oral surgery. Informed consent was obtained under a protocol approved by the Università Politecnica delle Marche (Ancona, Italy). Immediately after extraction, the central part of the dentai papilla was cut into small pieces, washed in phosphate buffered saline (PBS), and incubated in Dulbecco's modified Eagle's medium (DMEM) $/ \mathrm{F} 12$, containing $10 \%$ fetal bovine serum (FBS), $1 \%$ penicillin and streptomycin, and $1 \%$ fungizone. Monolayer cultures were maintained at $37^{\circ} \mathrm{C}$ in a humidified atmosphere of $5 \%(\mathrm{v} / \mathrm{v}) \mathrm{CO}_{2}$. Confluent HPFs were then detached with $0.25 \%$ trypsin in $1 \mathrm{mM}$ EDTA (Sigma-Aldrich). For cytotoxicity tests, cells were used between the $3^{\text {rd }}$ and $5^{\text {th }}$ passage of culture.

\section{DBSs preparation}

Polymerised resin disks were obtained using i) the bonding agent of a self-etch DBS (SE-DBS, Techbond, Isasan, Rovello Porro, Italy; $n=12$ ); and (ii) the primer agent of a two-step etch-and-rinse DBS (ER-DBS, Optibond Solo, Sybron-Kerr, Orange, CA; $n=12$ ). The liquid co-monomer mixtures were placed in silicone moulds $2 \mathrm{~mm}$ deep and $6 \mathrm{~mm}$ in diameter. The moulds were placed on a Mylar film-covered glass slide covered with another Mylar film glass slide, and light-cured on each side for $40 \mathrm{~s}$ using a quartz-tungsten-halogen unit at $600 \mathrm{~mW} / \mathrm{cm}^{2}$. The disks were removed from the moulds and dry-polished to a thickness of $1 \mathrm{~mm}$ (14).

\section{Cell treatment}

For each experiment, $3.5 \times 10^{4} \mathrm{cell} / \mathrm{cm}^{2}$ HPFs were incubated for $24 \mathrm{~h}$ with DMEM/F 12 medium conditioned as follows: group 1, DMEM/F12 maintained for $24 \mathrm{~h}$ (control 1); group 2, DMEM/F12 maintained for $96 \mathrm{~h}$ (control 2); group 3, three polymerised disks of SE (selfetch)-DBS immersed in culture medium for $24 \mathrm{~h}$; group 4 , three polymerised disks of ER (etch-and-rinse)-DBS immersed in the culture medium for $24 \mathrm{~h}$; group 5, three polymerised disks of SE-DBS immersed in the culture medium for $96 \mathrm{~h}$; and group 6, three polymerised disks of ER-DBS immersed in the culture medium for $96 \mathrm{~h}$. All experiments were performed in triplicate.

\section{MTT assay}

The effects of the two DBSs on HPF viability were determined using the 3-dimethylthiazol-2, 5-diphenyltetrazolium bromide (MTT) assay. After incubation for $24 \mathrm{~h}$ with the control or conditioned medium, HPFs were analysed. The media were removed, and $200 \mu \mathrm{L}$ MTT solution (Sigma-Aldrich, Milan, Italy; 5 $\mathrm{mg} / \mathrm{mL}$ in DMEM without phenol red) and $1.8 \mathrm{~mL}$ medium were added to the cell monolayers. After incubation at $37^{\circ} \mathrm{C}$ for $4 \mathrm{~h}$, the growth medium was removed, and $2 \mathrm{~mL}$ of solvent ( $1 \mathrm{~N} \mathrm{HCl}$ diluted to $4 \%$ in absolute isopropyl alcohol; Sigma) was added to each well to dissolve the purple crystals of formazan that had formed. Spectrophotometric quantification was then performed at $570 \mathrm{~nm}$ (Secomam, Anthelie light, version 3.8, Contardi, Italy). Results are expressed as the percentage absorbance over the control culture value. Experiments were run in triplicate and statistical analysis was performed using a Student's $t$-test.

Sodium dodecyl sulphate polyacrylamide gel electrophoresis (SDS-PAGE) and Western blotting

HPFs exposed to the control or DBSsconditioned culture media were lysed in modified radioimmunoprecipitation assay lysis buffer $(50 \mathrm{mmol} / \mathrm{L}$ Tris- $\mathrm{HCl}, \mathrm{pH}=7.4,1 \%$ Nonidet-P40, $150 \mathrm{mmol} / \mathrm{L}$ $\mathrm{NaCl}, 2 \mathrm{mmol} / \mathrm{L}$ EDTA, $0.1 \%$ sodium dodecylsulfate, 
$1 \mathrm{mmol} / \mathrm{L}$ ethylene-glycol-tetraacetic acid, $1 \mathrm{mmol} / \mathrm{L}$ phenylmethane-sulfonylfluoride, $\quad 0.15 \% \quad$ betamercaptoethanol) supplemented with $25 \mathrm{mmol} / \mathrm{L}$ protease inhibitor cocktail (Sigma-Aldrich). Total proteins were resolved by $8 \%$ SDS-polyacrylamide gel electrophoresis (SDS-PAGE) and electrophoretically transferred to a nitrocellulose membrane using a wet blotting apparatus (Mini Tank Electroblotting System, Owl, Portsmouth, UK). Membranes were blocked with $2.5 \%$ dry milk (Bebilac, Sicura, France) in TBS-Tween buffer (Tween $200.1 \%, \mathrm{NaCl} 0.15 \mathrm{~mol} / \mathrm{L}$, Tris-Base $0.01 \mathrm{~mol} / \mathrm{L}, \mathrm{pH}$ 7.5) for $1 \mathrm{~h}$ at room temperature and were labelled with goat anti-human procollagen $\alpha$ l type I primary antibody (Santa Cruz Biotechnology, Inc., Santa Cruz, CA) diluted 1:5000 for $2 \mathrm{~h}$ at $37^{\circ} \mathrm{C}$, and anti- $\beta$ tubulin antibody (Sigma Aldrich, Saint Luis, Missouri) diluted 1:10000, for $2 \mathrm{~h}$ at $37^{\circ} \mathrm{C}$ as positive loading control.

After three washes in TBS-Tween buffer, the membranes were labelled with horseradish peroxidase conjugated secondary antibodies (Santa Cruz Biotechnology, Inc. for procollagen $\alpha 1$ type I, and Sigma Aldrich, Saint Luis, Missouri, for $\beta$-tubulin), diluted 1:80,000, for $90 \mathrm{~min}$ at $37^{\circ} \mathrm{C}$. Positive bands were detected using the Enhanced Chemiluminescence System (ECL plus, Amersham Biosciences, Little Chalfont, UK). Images were obtained using an Image Station 2000R (Kodak, NY, USA).

\section{RESULTS}

\section{Human pulp fibroblast culture}

A primary culture of HPFs was established from the core of the dental pulp from the extracted healthy molars. Cells appeared near the explants after 7 days of culture (Fig. IA). After 14 days, the adherent cells began to divide rapidly, becoming a confluent and morphologically homogeneous cell population within the next 7 days (Fig. 1B).

\section{MTT assay}

The MTT test showed a significant $(p<0.05)$ inhibition of cell viability following treatment with the culture media conditioned for $24 \mathrm{~h}$ by polymerised disks of SE-DBS (group 3) and ERDBS (group 4), with percentages of viable cells of $45 \%$ and $30 \%$, respectively (significantly lower than control cells; Fig. 2). Treatment of HPFs with the culture media conditioned for $96 \mathrm{~h}$ with polymerised disks of SE-DBS (group 5) and ER-DBS (group 6) showed a significant decrease in inhibitory effects, with the percentage of viable cells being $62 \%$ and $77 \%$, respectively (Fig. 2 ).

\section{SDS-PAGE and Western blot}

Western-blot analysis showed intense expression of procollagen $\alpha 1$ type I, demonstrated by a strong band at approximately $250 \mathrm{kDa}$ for untreated control specimens at 24 and $96 \mathrm{~h}$ (groups 1 and 2; Fig. 3A, lanes 1 and 2), whereas faint bands at approximately $250 \mathrm{kDa}$ were seen with specimens exposed to both DBSs for $24 \mathrm{~h}$, corresponding to decreased synthesis of procollagen $\alpha 1$ type I in groups 3 and 4 (Fig. 3A, lanes 3 and 4, respectively). After $96 \mathrm{~h}$ of incubation, the DBS-treated specimens showed no expression of procollagen al type I, regardless of the DBS used (groups 5 and 6; Fig. 3A, lanes 5 and 6).
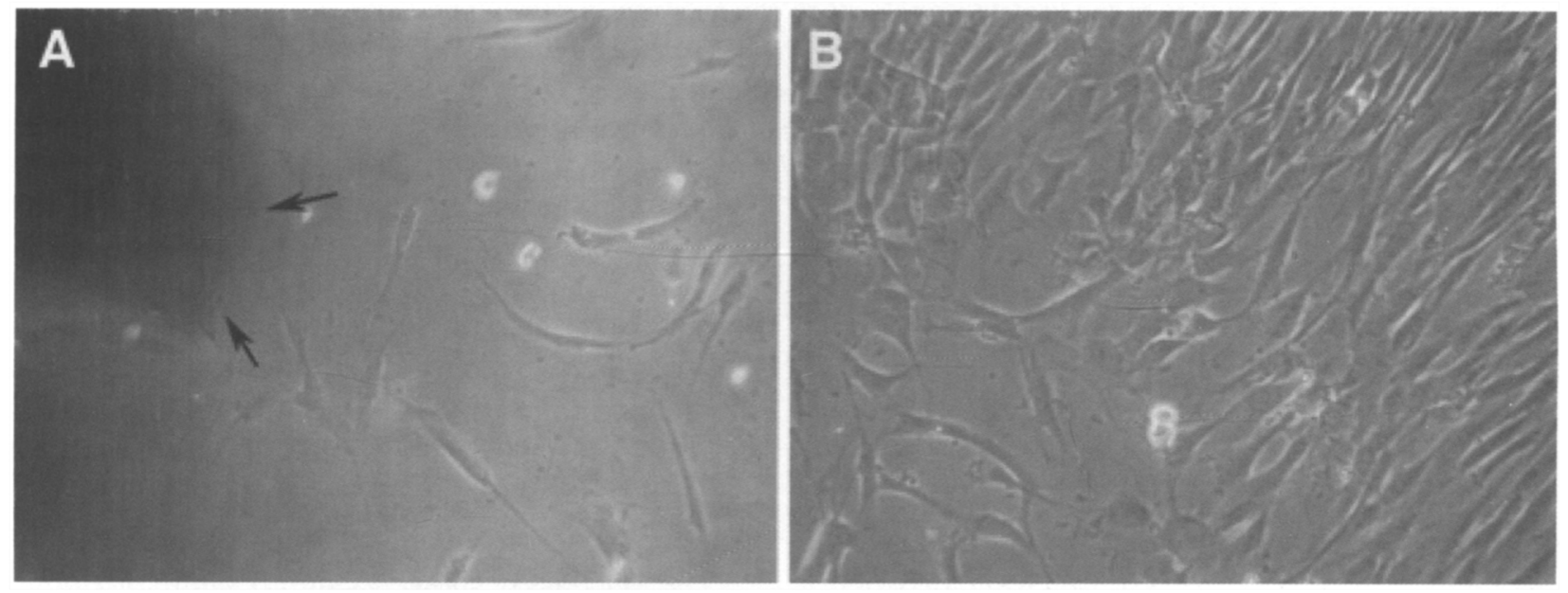

Fig. 1. Phase contrast microscopy images of the HPF culture: A) after 7 days of culture, there were early HPFs near the explant (arrows); B) after 21 days, the cell monolaver became confluent and homogeneous, with fibroblastoid morphology: 
MTT Viability TEST

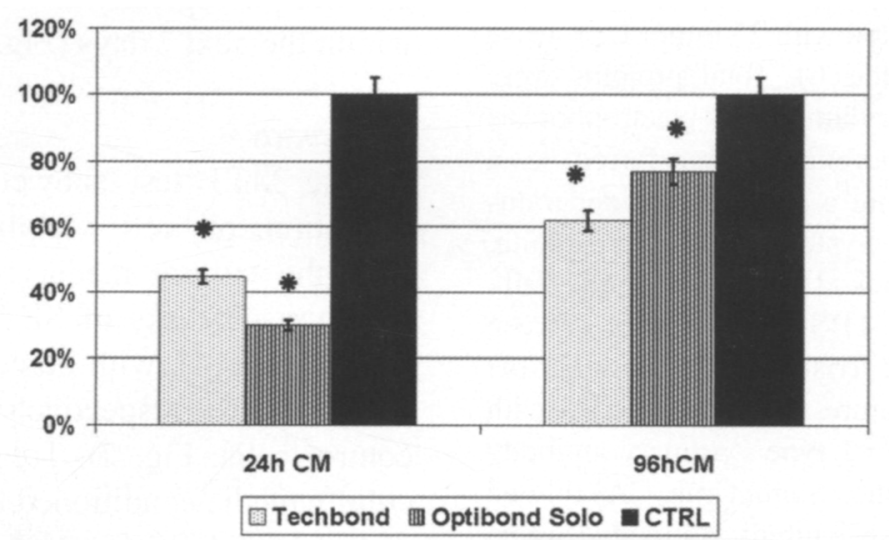

Fig. 2. Viability of HPFs after incubation with control culture medium and culture media conditioned by polymerised disks of the two tested DBSs (Techbond or Optibond Solo) for $24 \mathrm{~h}$ or $96 \mathrm{~h}$. Values are reported as relative percentages (y axis) measured in cells incubated with control medium (means \pm standard errors, from three experiments, each performed in triplicate). Asterisks indicate significant differences $(p<0.05)$ versus cells incubated with control medium (CTRL).

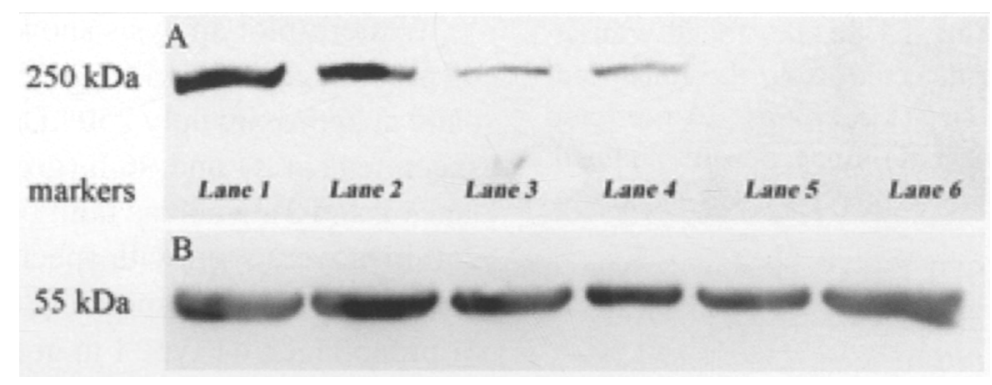

Fig. 3. Western blot analysis of HPFs extracts after incubation with control and DBS conditioned media. A) Expression of procollagen al type I. Lanes 1-2: procollagen al type I expression from HPFs incubated for $24 \mathrm{~h}$ in DMEM/FI2 previously maintained for 24 (control 1 - Lane 1) or $96 \mathrm{~h}$ in the incubator (control 2-Lane 2): a strong band at approximately 250 $k D a$ was detected in control 1 and 2. Lanes 3 and 4: procollagen al type I expression from HPFs incubated for $24 \mathrm{~h}$ with medium previously conditioned with the addition of polymerised disks of Techbond (Lane 3) or Optibond Solo (Lane 4) for $24 \mathrm{~h}$ showing a strong reduction in the protein signal, which can be visualized as a faint band at $250 \mathrm{kDa}$. These results were similar for both adhesives. Lanes 5-6: procollagen al type I expression from HPFs incubated for $24 \mathrm{~h}$ with media previously conditioned by adding polymerised disks of Techbond (Lane 5) or Optibond Solo (Lane 6) for $96 \mathrm{~h}$ revealing no band for either treatment. B) Western blot analysis of $\beta$-tubulin (loading control) in HPFs after incubation with control and DBSs conditioned media. $\beta$-tubulin expression confirmed similar protein loading in all lanes.

$\beta$-tubulin expression confirmed similar protein loading in all lanes (Fig. 3B).

\section{DISCUSSION}

The present study showed that HPFs treated with both tested DBSs (i.e., two-step self-etch and two-step etch-and-rinse) resulted in decreased HPF viability, which was more pronounced after $24 \mathrm{~h}$ than after
$96 \mathrm{~h}$, and decreased procollagen $\alpha \mathrm{l}$ type I expression, that was then abolished after exposure to the 96-h conditioned culture media. Thus, the null hypotheses tested were rejected because both DBSs tested and the different conditioning times affected i) HPFs viability and ii) procollagen $\alpha 1$ type I expression.

The MTT assay showed a significant reduction in cell viability after treatment with DBS-conditioned media, which was higher with 24-h-conditioned 
medium than with the 96-h-conditioned medium. This could be due to a possible reduction in monomers eluting over time in addition to their potential neutralization by the culture medium, suggesting that HPF alteration after exposure to the two tested DBSs may be not irreversible.

Of the two categories of DBSs tested in the present study [i.e., self-etch (SE-DBS) or etch-and-rinse (ER-DBS)], the SE two-step product showed a lower cytotoxic effect at $24 \mathrm{~h}$ than the simplified two-step ER product. In contrast, both DBSs induced increased cell viability at $96 \mathrm{~h}$, with the ER-DBS being less cytotoxic than the SE-DBS. These findings are consistent with those of Breschi et al., who showed that simplified DBS, such as the two-step ER-DBS, exhibited a lower extent of polymerization as compared with the multi-step SE approach (15). The higher percentage of hydrophilic monomers and the presence of water in the two-step ER-DBSs may compromise their polymerization, also leading to higher permeability (15). Expression of procollagen $\alpha 1$ type I protein was decreased in HPFs incubated with both DBS-24-hconditioned media versus cells cultured with control media. Procollagen al type I protein expression became undetectable when HPFs were incubated with the 96-h conditioned media of both DBSs. These data are consistent with previous studies that investigated the effects of different concentrations and times of exposure to HEMA on procollagen $\alpha 1$ type 1 expression, showing a decrease in the protein with an increase in exposure time (8-10).

Overall, the results of the present study support the hypothesis that monomer eluates of DBSs do not significantly affect HPF viability and metabolism, because recovered cellular viability was seen after $96 \mathrm{~h}$; however, leaching monomers may markedly decrease the synthesis of matrix extracellular proteins, such as procollagen $\alpha 1$ type I.

\section{ACKNOWLEDGEMENTS}

The authors report no conflict of interest and wish to thank Mr. Aurelio Valmori for extensive laboratory and photographical assistance and the manufacturers for kindly providing the materials. The study was partially founded with the following grants from MIUR (Italy): FIRB RBAP1095CR and PRIN 2009SAN9K5 P.I. Breschi L; FIRB RBAP10MLK7
P.I. Di Primio R.

\section{REFERENCES}

1. Goldberg M, Smith AJ. Cells and Extracellular Matrices of Dentin and Pulp: A Biological Basis for Repair and Tissue Engineering. Crit Rev Oral Biol Med 2004; 15:13-27.

2. Orsini G, Ruggeri A Jr, Mazzoni A, Papa V, Piccirilli M, Falconi M, Breschi L. Immunohistochemical identification of type I and type III collagen and chondroitin sulphate in human pre-dentine: a correlative FEI-SEM/TEM study. Int Endod J 2007; 40:669-78.

3. Shuttleworth CA, Berry L, Wilson N. Collagen synthesis in rabbit dental pulp fibroblast cultures. Arch Oral Biol 1980; 25:201-5.

4. Kuo MY, Lan WH, Lin SK, Tsai KS, Hahn LJ. Collagen gene expression in human dental pulp cell cultures. Arch Oral Biol 1992; 37:945-52.

5. Narayanan AS, Page RC. Biochemical characterization of collagens synthesized by fibroblasts derived from normal and diseased human gingiva. J Biol Chem 1976; 251:5464-71.

6. Bouillaguet $\mathrm{S}$, Virgillito $\mathrm{M}$, Wataha $\mathrm{J}$, Ciucchi $\mathrm{B}$, Holz $\mathrm{J}$ The influence of dentine permeability on cytotoxicity of four dentine bonding systems, in vitro. J Oral Rehabil 1998; 25:45-51.

7. Bouillaguet S, Wataha JC, Virgillito M, Gonzalez L, Rakich DR, Meyer JM. Effect of sub-lethal concentrations of HEMA (2-hydroxyethyl methacrylate) on THP-1 human monocytemacrophages, in vitro. Dent Mater 2000; 16:213-7.

8. Teti G, Mazzotti G, Zago M, Ortolani M, Breschi L, Pelotti S, Ruggeri A, Falconi M. HEMA downregulates procollagen alphal type $I$ in human gingival fibroblasts. J Biomed Mater Res A 2009; 90:256-62.

9. Falconi M, Ortolani M, Teti G, Zago M, Orsini G, Selan L, Mazzotti G. Suppression of Procollagen \{alpha\} 1 type 1 by long-term low-dose exposure to 2-hydroxyethylmethacrylate in human gingival fibroblasts in vitro. Int J Toxicol 2010; 29:523-31.

10. Zago M, Teti G, Mazzotti G, Ruggeri A, Breschi L, Pelotti S, Ortolani M, Falconi M.. Expression of procollagen alphal type I and tenascin proteins induced by HEMA in human pulp fibroblasts. 
Toxicol In Vitro 2008;22(5):1153-9.

11. Issa Y, Watts DC, Brunton PA, Waters CM, Duxbury AJ. Resin composite monomers alter MTT and LDH activity of human gingival fibroblasts in vitro. Dent Mater 2004; 20:12-20..

12. Geurtsen W, Lehmann F, Spahl W, Leyhausen G. Cytotoxicity of 35 dental resin composite monomers/ additives in permanent $3 \mathrm{~T} 3$ and three human primary fibroblast cultures. J Biomed Mater Res 1998; 41:474-80.

13. About I, Camps J, Burger AS, Mitsiadis TA, Butler
WT, Franquin JC. Polymerized bonding agents and the differentiation in vitro of human pulp cells into odontoblast-like cells. Dent Mater 2005 ;21:156-63.

14. Orsini G, Mazzoni A, Orciani $M$, et al. Matrix metalloproteinase-2 expression induced by two different adhesive systems on human pulp fibroblasts. J Endod 2011; 37:1663-7.

15. Breschi L, Cadenaro M, Antoniolli F, Visintini E, Toledano M, Di Lenarda R. Extent of polymerization of dental bonding systems on bleached enamel. Am J Dent 2007; 20:275-80. 